\section{A propósito de la contingencia COVID-19. ECMO en el adulto: Oxigenación por membrana extracorpórea. A quién, cómo y cuándo}

\author{
RODRIGO DÍAZ ${ }^{1,5}$, MARTA VELIA ANTONINI 2,5,a, \\ RODRIGO ORREGO ${ }^{3}$, DARRYL ABRAMS ${ }^{4,5}$
}

\section{With regard to COVID-19 contingency. ECMO in adults. Extracorporeal Membrane Oxygenation). To whom, how and when}

ECMO (Extracorporeal Membrane Oxygenation) is an extracorporeal life support system in catastrophic lung failure, shock and cardiopulmonary resuscitation, in different age groups, with multiple physiologic features. When the candidate to be submitted is too unstable to be transported to a hospital with ECMO, cannulation before transfer allows stabilization and subsequent transport. The aim of this article is to review the current concepts of extracorporeal support, its indications, national and international experience, and its possible role in the SARS-Cov2 pandemic.

(Rev Med Chile 2020; 148: 349-361)

Key words: Extracorporeal Membrane Oxygenation; Respiratory Distress Syndrome, Adult; Shock, Cardiogenic.

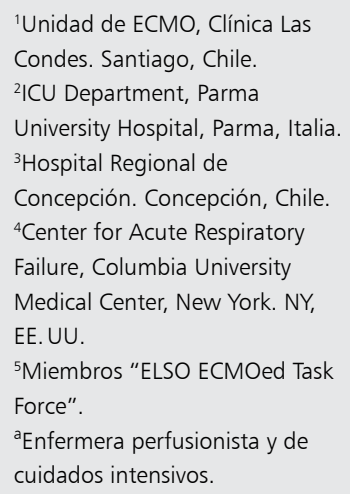

Recibido el 16 de abril de 2020, aceptado el 6 de mayo de 2020

Correspondencia a: Rodrigo Díaz Gómez Estoril 450, Clínica Las Condes. Santiago, Chile. diazrodrigo@me.com
$\mathrm{E}$ n 1971 JD Hill publicó el primer uso de un circuito extracorpóreo para uso prolongado, con sobrevida del paciente. Conectó a un sistema de oxigenación extracorpórea por membrana (ECMO por sus siglas en inglés) a un joven de 24 años con un distrés respiratorio secundario a politraumatismo ${ }^{1}$. En 1972 Bartlett reportó el primer ECMO cardiaco en un niño post cirugía cardiaca, y en 1975 el primer caso neonatal ${ }^{1}$. En 1979, el fracaso del primer estudio aleatorizado de ECMO del National Institutes of Health de EE.UU. ${ }^{2}$ provocó una paralización del desarrollo ECMO para la asistencia en adultos, quedando su uso casi exclusivamente en el ambiente neonatal, hasta el año 2009, en que ocurrió la pandemia por Influenza H1N1 y fue publicado el estudio CESAR (Conventional Ventilatory Support vs Extracorporeal Membrane Oxygenation for Severe Adult Respiratory Failu$r e)^{3}$, que fue aleatorizado y el primero que aportó evidencia sólida sobre el uso y beneficios del uso de este sistema de oxigenación con las máquinas y conceptos actuales. Desde entonces el uso de ECMO ha aumentado en todas sus indicaciones: falla respiratoria, cardiaca o como asistencia en paro cardiorespiratorio.

El intercambio de gases transmembrana extracorpórea o ECMO utiliza una bomba y un oxigenador (intercambiador de gases) para proveer soporte hemodinámico y/o respiratorio prolongado. Dependiendo del tipo de paciente es el tipo de ECMO que se elige: veno venoso para la falla respiratoria y/o venoarterial cuando existe compromiso hemodinámico. El ECMO realiza intercambio de gases en la membrana del "oxigenador", y el soporte hemodinámico lo hace en su configuración veno arterial drenando el lado derecho de la circulación y devolviendo en el lado sistémico ya sea en una arteria femoral, arteria axilar o directamente en la aorta (Figura 1). 


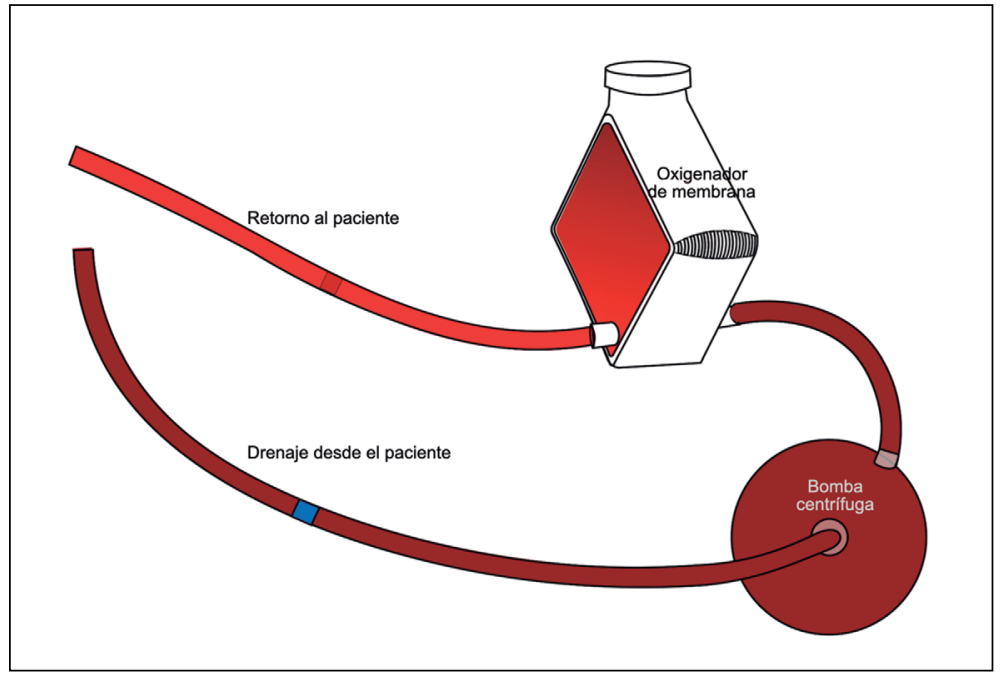

Figura 1. Sistema de ECMO convencional. Línea desde el paciente, bomba centrífuga, membrana de intercambio de gases y línea de retorno al paciente.
Hace dos años, ELSO (Extracorporeal Life Support Organization), propuso una nueva nomenclatura ${ }^{4,5}$, lo que ordena algunas definiciones, dejando la extracción extracorpórea de $\mathrm{CO}^{2}$ (ECCO2R por su siglas en inglés), fuera del término ECMO (Figura 2). En este cuadro se describen las indicaciones actuales de los tipos de ECMO, destacando que para las fallas respiratorias la elección es la canulacion veno venosa (ECMO VV) y para la insuficiencia cardiaca aguda es la canulacion veno arterial (ECMO VA).

Desde hace 3 décadas los resultados de esta técnica son consignados en la base de datos ELSO, que cuenta actualmente con más de cien mil regis-

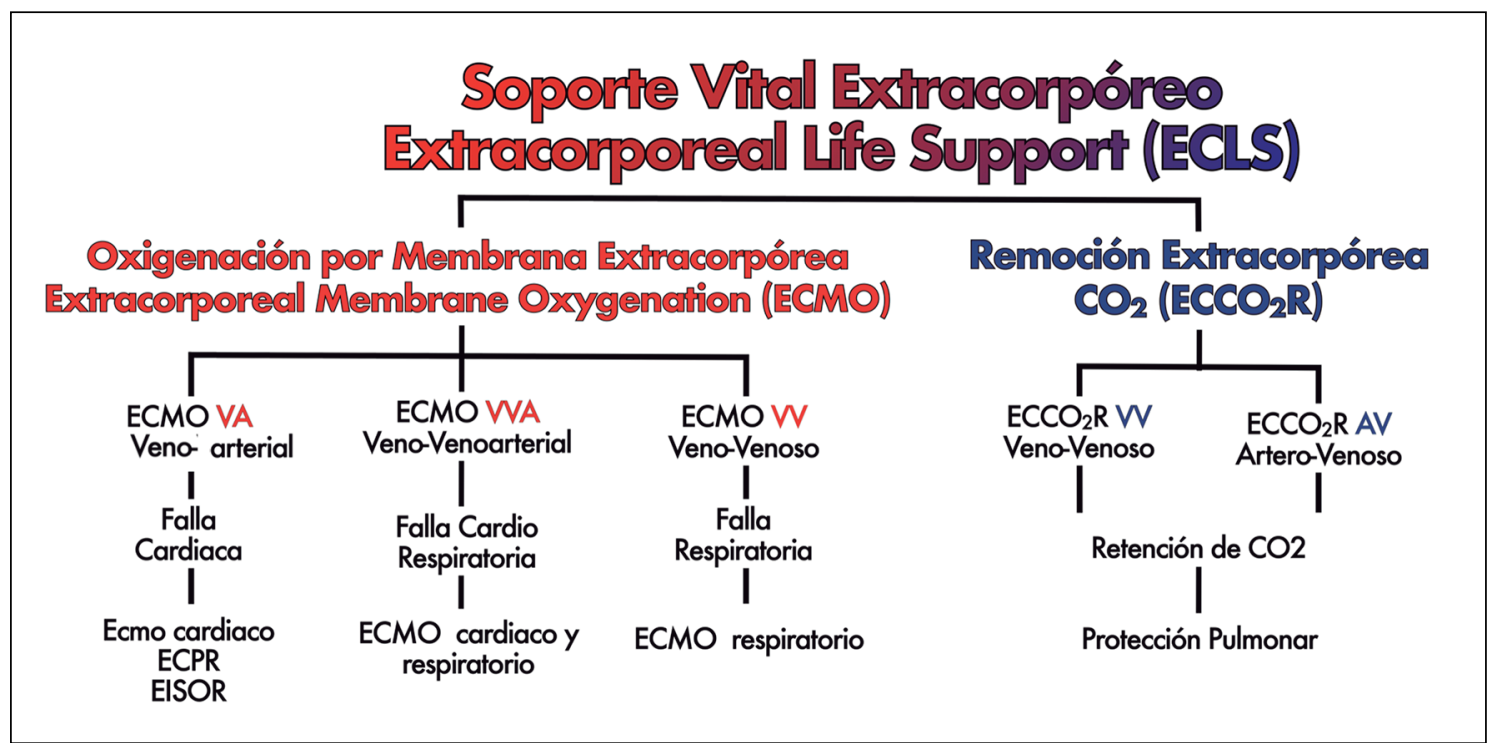

FIGURA 2. Relación sistema-modo de soporte e indicacion de ECLS. VA: veno arterial; VVA: venovenoarterial; VV: veno venoso; $\mathrm{ECCO}_{2} \mathrm{R}$ : remoción $\mathrm{CO}_{2}$ extracorpórea; AV: arterio venosa; ECPR: reanimación cardiopulmonar extracorpórea; EISOR: extracorporeal interval support for organ retrieval. Reprinted with permission of the American Thoracic Society. Copyright ${ }^{\circ} 2019$ American Thoracic Society. Conrad S et al. American journal of respiratory and critical care medicine 198 (4): 447-451. The American Journal of Respiratory and Critical Care Medicine is an official journal of the American Thoracic Society. 
tros de pacientes de todo el mundo. Seis centros en Chile aportan con casos activamente desde el 2009 y, en conjunto, Latinoamérica ha aportado aproximadamente $1 \%$ del total de registros a la fecha (Tabla 1) (Fuente Registro ELSO).

El objetivo de esta revisión, es describir los conceptos actuales del soporte extracorpóreo, sus indicaciones, experiencia nacional e internacional, y su posible rol en la pandemia por SARS-Cov2.

\section{Indicaciones}

\section{Falla respiratoria catastrófica}

El ensayo EOLIA (ECMO to Rescue Lung Injury in Severe ARDS), publicado en 2018, no mostró una diferencia estadisticamente significativa de mortalidad a los 60 días en Distress Respiratorio Severo (SDRA), pero sí una tendencia importante a favor del ECMO, con mucho cruce de pacientes del grupo control a soporte ${ }^{7}$, si bien los resultados son controversiales en su interpretación ${ }^{8}$, el consenso en general, es que la decisión es caso a caso9.

La indicación más frecuente de ECMO veno venoso (VV) es la falla respiratoria catastrófica ${ }^{10}$, la cual tiene varias definiciones (ejemplo: escala de
Murray $>3$ ), que tienen como factor común, el fracaso a las medidas convencionales más avanzadas. La demora en la decisión de iniciar el soporte se asocia a una mayor mortalidad ${ }^{11}$. Sin embargo, antes de indicarlo, es necesario cumplir con los siguientes pasos:

1. Identificar causa de la falla respiratoria.

2. Que el paciente esté en ventilación mecánica protectora con PEEP alto, pero manteniendo una presión diferencial $<15 \mathrm{~cm} \mathrm{H}_{2} \mathrm{O}^{12,13}$.

3. Restricción de fluidos ${ }^{14}$.

4. Bloqueo neuromuscular ${ }^{15}$.

5. Prueba de posición prono (se sugiere $6 \mathrm{~h}$ post prono reevaluar mecánica pulmonar y relación $\left.\mathrm{PaFiO}_{2}\right)^{16,17}$.

6. Cumplido lo anterior, considerar ECMO, según los siguientes criterios:

a) Paciente hipoxémico con compliance severamente disminuida (Tabla 2 ).

b) Hipercapnea sostenida (grupo de mayor beneficio del ECMO en disminución de mortalidad según el estudio EOLIA $)^{18}$.

c) Otras Indicaciones como fuga aérea masiva $\left(>50 \%\right.$ del Volumen Corriente) ${ }^{19}$.

d) Contraindicaciones relativas. Criterio conservador extraído de estudio EOLIA²:

Tabla 1. Registro Internacional ELSO Enero 2020

\begin{tabular}{|c|c|c|c|c|c|}
\hline \multirow{2}{*}{ Enero 2020} & \multicolumn{5}{|c|}{ Reporte del Registro, Resultados - Resumen Internacionc } \\
\hline & Total casos & \multicolumn{2}{|c|}{ Salida de ECMO } & \multicolumn{2}{|c|}{$\begin{array}{l}\text { Sobrevida al } \\
\text { alta o traslado }\end{array}$} \\
\hline \multicolumn{6}{|l|}{ Neonatal } \\
\hline Respiratorio & 32285 & 28417 & $87 \%$ & 23675 & $73 \%$ \\
\hline Cardiaco & 8830 & 6097 & $69 \%$ & 3818 & $43 \%$ \\
\hline ECPR & 2035 & 1427 & $70 \%$ & 861 & $42 \%$ \\
\hline \multicolumn{6}{|l|}{ Pediatrico } \\
\hline Respiratorio & 10346 & 7471 & $72 \%$ & 6199 & $59 \%$ \\
\hline Cardiaco & 12538 & 9042 & $72 \%$ & 6667 & $53 \%$ \\
\hline ECPR & 4945 & 2940 & $59 \%$ & 2086 & $42 \%$ \\
\hline \multicolumn{6}{|l|}{ Adulto } \\
\hline Respiratorio & 24395 & 16971 & $69 \%$ & 14714 & $60 \%$ \\
\hline Cardiaco & 25488 & 15184 & $59 \%$ & 11191 & $43 \%$ \\
\hline ECPR & 8075 & 3363 & $41 \%$ & 2387 & $29 \%$ \\
\hline Total & 129037 & 90912 & $70 \%$ & 71598 & $55 \%$ \\
\hline
\end{tabular}

Fuente: International Summary -Jan, 2020. Extracorporeal Life Support Organization 2800 Plymouth Road Building 300, Room 303 Ann Arbor, MI 48109. 
Tabla 2. Criterios de Ingreso a ECMO en falla respiratoria catastrófica en SDRA

\begin{tabular}{|c|c|c|c|}
\hline & 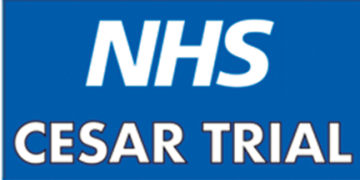 & $\begin{array}{l}\text { ASSISTANCE } \\
\text { PUBLIQUE } \\
\text { HOPITAUX }\end{array}$ & SOCHIMIt*t \\
\hline 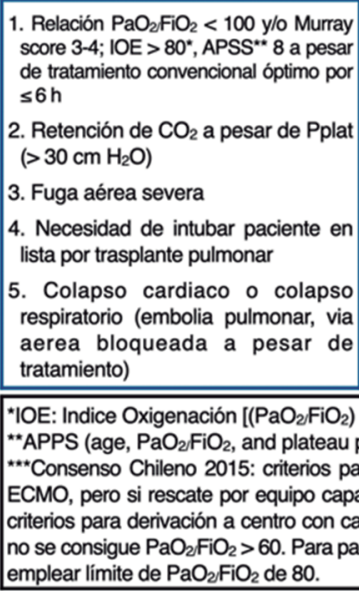 & $\begin{array}{l}\text { Falla respiratoria potencialmente } \\
\text { reversible Murray }>3.0 \\
\mathrm{pH}<7.20 \text { a pesar de tratamiento } \\
\text { convencional adecuado }\end{array}$ & $\begin{array}{l}\text { 1. Relación } \mathrm{PaO}_{2} \mathrm{FiO}_{2}<50 \text { con } \mathrm{FiO}_{2} \\
>0.8 \text { por }>3 \mathrm{~h} \\
\text { 2. Relación } \mathrm{PaO}_{2} \mathrm{FiO}_{2}<80 \text { con } \mathrm{FiO}_{2} \\
>0.8 \text { por } 6 \mathrm{~h} \\
\text { 3. } \mathrm{pH}<7.25 \text { por } 6 \mathrm{~h} \text { con } \mathrm{FR} 35 / \mathrm{min} \\
\text { y VM con Pplateau }<32 \mathrm{~cm} \mathrm{H} \mathrm{O}_{2}\end{array}$ & $\begin{array}{l}\text { Paciente con causa reversible con } \\
\text { Murray }>2.5 \text { y VM protectora (Vt } 6 \\
\text { m/kg ideal) con presión diferencial < } \\
15 \mathrm{~cm} \mathrm{H} \mathrm{H}_{2} \mathrm{O} \text { con PEEP optimizado. } \\
\text { Insuficiencia respiratoria grave que } \\
\text { no responde a: } \\
\text { Prueba o ensayo con bloqueo } \\
\text { neuromuscular en infusión } \\
\text { continúa. } \\
\text { Prueba o ensayo de VM en } \\
\text { posición prono. } \\
\text { a. } \mathrm{PaO}_{2} \mathrm{FiO}_{2}<100 \text { persistente por } 4-6 \\
\text { horas o } \mathrm{PaO}_{2} \mathrm{FiO}_{2}<60 \text { por } 2 \text { horas. } \\
\text { b. pH }<7.0 \text { y } \mathrm{PaCO}_{2}>100 \text { por } 6 \\
\text { horas. } \\
\text { Indicación excepcional: } \\
\text { paciente con diagnóstico de HANTA, } \\
\text { independiente de sus condiciones } \\
\text { de oxigenación. Se traslada } \\
\text { precozmente a centro con capacidad } \\
\text { de ECMO. }\end{array}$ \\
\hline
\end{tabular}

Índice oxigenación: $100 \times \mathrm{FiO}_{2} \times$ (Presión plateau/ $\mathrm{PaO}_{2}$ ). IOE (Índice oxigenación + edad): $1 \mathrm{O}+$ edad (años). APPS (age, PaO $/$ $\mathrm{FiO}_{2}$, and plateau pressure score).

- Ventilación mecánica por 7 días o más.

- Peso de más de $1 \mathrm{~kg}$ por centímetro de altura o un índice de masa corporal $>45$.

- Insuficiencia respiratoria crónica de larga data, con oxígeno domiciliario o ventilación no invasiva.

- Antecedentes de trombocitopenia inducida por heparina.

- Cáncer con una esperanza de vida de menos de 5 años.

- Condición moribunda o un valor de puntuación de fisiología aguda simplificada (SAPS-II) de más de 90 (0 a 163).

- Coma actual después de un paro cardíaco, no inducido por drogas.

- Lesión sistema nervioso central irreversible.

La mortalidad en ECMO en pacientes con falla respiratoria catastrófica tiene determinantes como: edad, días previos de ventilacion mecánica, falla de órganos, etiología de la patología pulmonar y el estado inmunológico, que deben tomarse en cuenta antes de indicar su uso ${ }^{20}$. En la Tabla 3 se aprecian las sobrevidas observadas en distintas series.
Puntos clave: Usualmente conexión VV, criterio EOLIA es el más utilizado, pero se reconoce que la selección de los pacientes es caso a caso.

\section{Otras aplicaciones respiratorias}

1. Puente de pacientes a trasplante pulmonar (Bridge To Transplant, BTT por sus siglas en inglés) (Figura 3$)^{20,21}$.

2. Otras formas de hipoxemia refractaria o insuficiencia respiratoria hipercapnica ${ }^{20,22}$.

3. Tumores, malformaciones o cuerpos extraños y otras emergencias de la vía aérea ${ }^{23}$.

En el caso del BTT, la movilizacion y reacondicionamiento físico se ven facilitados por el ECMO, y son una variable muy importante en el éxito del trasplante ${ }^{21}$.

Puntos clave de ECMO Brigde to Transplant (BTT) y otras aplicaciones respiratorias:

1. Conexión a ECMO VV o ECMO VA.

2. El puente a trasplante ofrece la posibilidad de llevar el injerto a pacientes que se deterioran en su espera mientras estan en lista de espera. 
Tabla 3. Series de pacientes ECMO VV por falla respiratoria

\begin{tabular}{|c|c|c|c|}
\hline \multicolumn{4}{|c|}{ Series de } \\
\hline Autor & Año & \# pacientes & Sobrevida \\
\hline Zapol & 1979 & 42 & 0.1 \\
\hline Gattinoni & 1986 & 43 & 0.49 \\
\hline Peek & 1997 & 50 & 0.66 \\
\hline Mols & 2000 & 62 & 0.55 \\
\hline Linden & 2000 & 15 & 0.76 \\
\hline Hemmila et al & 2004 & 252 & 0.52 \\
\hline Brogan & 2009 & 1473 & 0.50 \\
\hline CESAR & 2009 & 180 & 0.63 \\
\hline ANZ ECMO & 2009 & 68 & 0.71 \\
\hline PRESERVE & 2013 & 140 & 0.64 \\
\hline CLC & 2019 & 146 & 0.63 \\
\hline EOLIA & 2018 & 124 & 0.65 \\
\hline
\end{tabular}

CLC = Clínica Las Condes.

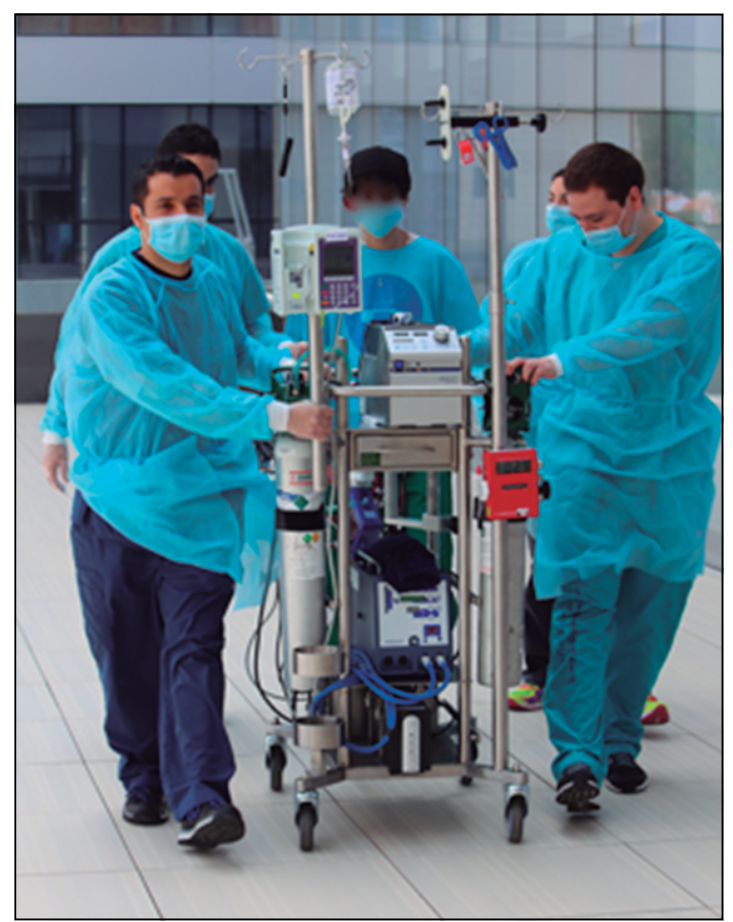

Figura 3. Paciente en puente a trasplante bipulmonar por hipertensión pulmonar post capilar (con permiso).

\section{Shock cardiogénico}

En las descripciones del shock cardiogénico (SC) siempre hay dos componentes básicos: hipotensión e hipoperfusión tisular (confusión mental, mareos, extremidades frías, oliguria, presión de pulso disminuida, acidosis metabólica, lactato y creatinina elevados) ${ }^{24}$. La mayor causa (aproximadamente $80 \%$ ) es la cardiopatía isquémica, seguida por insuficiencia cardiaca crónica descompensada $(11 \%)$, disfunciones valvulares y otras causas mecánicas $(6 \%)$, miocarditis (2\%) y cardiomiopatía inducida por estrés $(2 \%)^{25,26}$.

Dado que la mortalidad está asociada a la gravedad de los trastornos hemodinámicos ${ }^{27}$, es importante poder estratificar el riesgo de estos pacientes, para así considerar, como en todas las otras indicaciones de ECMO, una adecuada relación riesgo vs beneficio. La clasificacion de la Sociedad de Angiografia Intervencional da cuenta de esto (Figura 4).

En etapa refractaria, es decir cuando la hipotensión e hipoperfusión no responden al tratamiento médico (con o sin uso de balon de contrapulsación), el soporte circulatorio temporal (SCT) puede revertir esta situación, es así como distintas guías recomiendan uso de soporte extarcorpóreo en este escenario ${ }^{28-30}$.

El ECLS veno-arterial proporciona soporte respiratorio y cardiaco, entregando el flujo sanguíneo sistémico necesario a órganos vitales de pacientes en shock, mientras se revierte la disfunción cardia$\mathrm{ca}$, o como puente a otras alternativas. El ECMO podría estar asociado a mejores resultados en este contexto $^{31}$ y se considera de "primera línea" ya que la canulación vascular es relativamente sencilla, proporciona flujos altos, y a un costo menor que otros dispositivos ${ }^{30}$.

Una publicación reciente, que establece un enfoque estandarizado basado en equipos (shock team), ha mostrado una mejoria de los resultados del ratamiento del SC, aumentando significativamente la supervivencia a 30 días desde $47 \%$ a $76,6 \%{ }^{32}$. El reconocimiento rápido, la monitorización avanzada, el trabajo en equipo, las estrategias de reperfusión adecuadas y la indicación temprana de soporte circulatorio mecánico son esenciales para mejorar la sobevida en el shock cardiogénico. Nunca olvidar que el ECMO en falla cardiaca es una excelente herramienta para mantener el flujo a los órganos terminales, pero es un soporte que puede aumentar severamente la postcarga del 


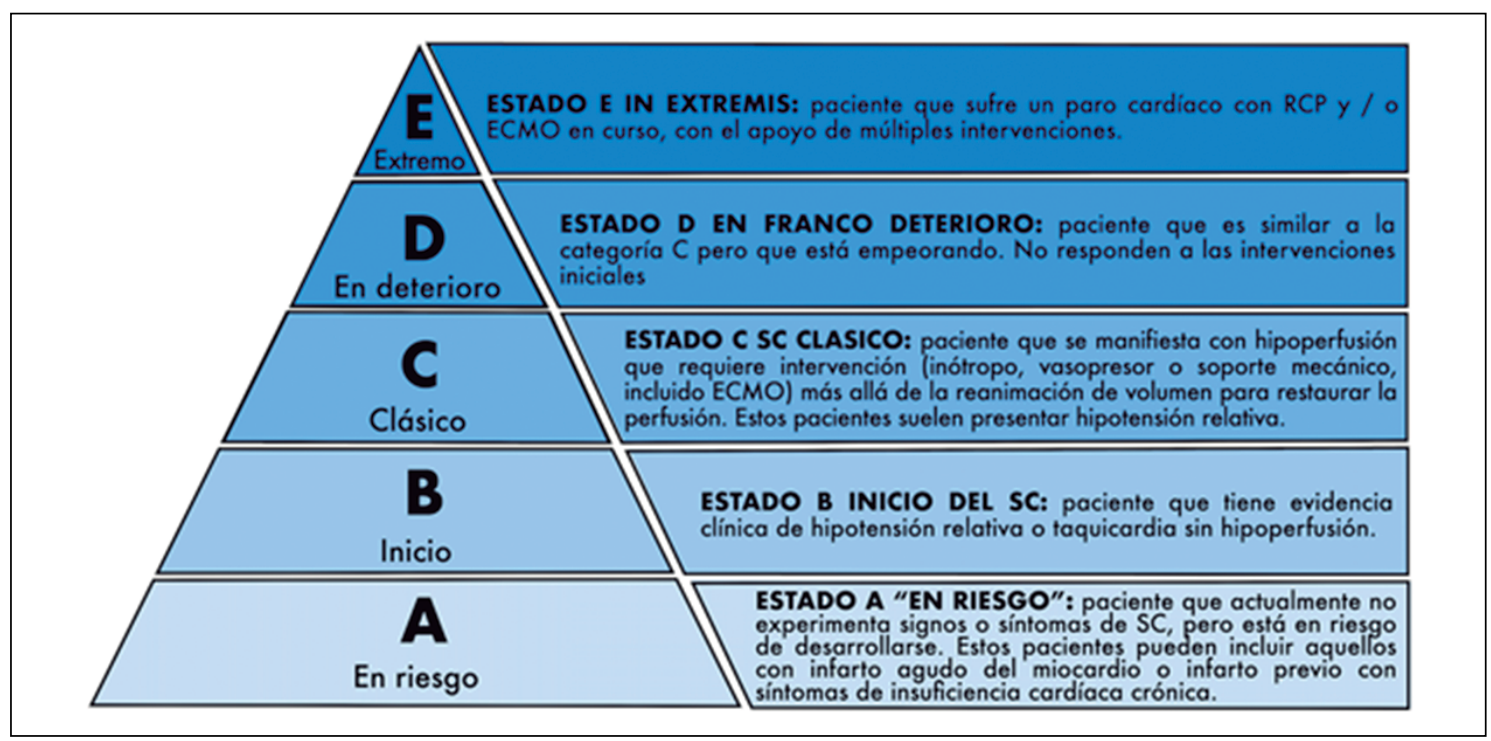

Figura 4. Clasificación del shock cardiogénico de la SCAI (Society for Cardiovascular Angiography and Intervention). RCP: Reanimación cardiopulmonar; ECMO: Oxigenación con membrana extracorpórea; SC: Shock Cardiogénico. Baran, David A., et al. SCAI clinical expert consensus statement on the classification of cardiogenic shock. Catheterization and Cardiovascular Interventions 2019; 94 (1): 29-37. Con permiso: John Wiley and Sons.

ventrículo izquierdo y comprometer su viabilidad, por lo que es un punto que hay que monitorizar periodicamente e intervenir precozmente de ser necesario ${ }^{33}$.

Puntos clave: Conexión VA central o periférica. El umbral de indicación es el shock refractario antes de que el paciente presente daño irreversible en órganos terminales.

\section{Sepsis}

Su uso en shock séptico es controversial en adultos, las series publicadas son escasas y no existen estudios aleatorizados de ECLS en esta condición. La sobrevida del ECMO VA es más baja en pacientes sépticos vs no sépticos, pero parece haber una diferencia relevante en resultados si existe o no falla ventricular, siendo paradójicamente más alta cuando esta está presente, quizás porque es en el escenario de hipotensión y mala perfusión por falla de bomba, donde fisiológicamente el ECMO tiene un rol, al sustituir temporalmente la función del corazón.

Huang et al sugieren un corte de edad a los 60 años $^{37}$. Park y Cheng identifican en analisis mul- tivariado, que el paro en shock séptico determina una mayor mortalidad (al punto que es una fuerte contraindicacion para ECLS en este contexto $)^{34,35}$. Factores asociados a mayor sobrevida, que se repiten en distintas series son: foco pulmonar, ECMO VV, NO RCP previa, menor tiempo desde la sepsis a la conexion del ECMO, función ventricular anormal o biomarcadores de daño miocardicos en valores elevados (Tabla 4).

Aunque se necesitan más datos y estudios al respecto, el ECLS parece tener un lugar en:

1. Disfunción pulmonar severa y shock séptico, con sobrevida alrededor de $70 \%$ (indicación es ECMO VV) ${ }^{38}$.

1. Shock séptico con disfunción uni o biventricular y deuda de oxigeno persistente que no responde a medidas habituales (indicación es ECMO VA), con sobrevida alrededor de $70 \%$. Cuando el patrón hemodinámico es puramente de hipotensión dada por vasoplegia, el ECMO no tiene buen resultado en esa población ${ }^{39}$, aunque la serie publicada por el grupo sueco en el 2019 muestra resultados aceptables incluso en esa población. Sin embargo hay que considerar que este grupo tiene una gran experiencia y equipos muy bien entrenados (pacientes conec- 
Tabla 4. Series de ECMO en shock séptico

\section{Series de ECMO en Shock Septico}

\begin{tabular}{|c|c|c|c|c|c|c|c|}
\hline 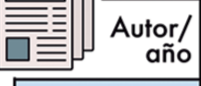 & Ciudad/Pais & $\begin{array}{c}\text { ECMO } \\
\text { configuración }\end{array}$ & $\mathrm{n}$ & Edad & $\begin{array}{l}\text { Paro PRE } \\
\text { ECMO }\end{array}$ & Foco Infección & $\begin{array}{c}\text { Sobrevida } \\
\text { al alta }\end{array}$ \\
\hline $\begin{array}{l}\text { Brechot } \\
\text { (2013) }\end{array}$ & Paris, Francia & VA periferico & 14 & $45(28-66)$ & $0 \%$ & Neumonia $(79 \%)$ & $71 \%$ \\
\hline Park (2015) & Seul, Corea & VA periferico & 32 & 55 & $44 \%$ & Neumonia $(34.4 \%)$ & $22 \%$ \\
\hline Cheng (2016) & Taipei, Taiwan & $\begin{array}{l}\text { VA } 80 \% \\
\text { VV } 20 \%\end{array}$ & 151 & $51(17-87)$ & $25 \%$ & Neumonia $(50.3 \%)$ & $30 \%$ \\
\hline Von Bar (2017) & Estocolmo, Suecia & $\begin{array}{l}\text { VA } \\
\text { VV 68\% }\end{array}$ & 255 & $46(33-58)$ & $0 \%$ & Neumonia $(72 \%)$ & $64 \%$ \\
\hline Vogel (2018) & Londres, UK & VA periferico & 12 & $40.5(23.7-50)$ & $42 \%$ & Neumonia (83\%) & $75 \%$ \\
\hline Ro (2018) & Seul, Corea & VA periferico & 71 & $56.0 \pm 12.3$ & $12 \%$ & Neumonia $(70 \%)$ & $7 \%$ \\
\hline Falk (2019) & Estocolmo, Suecia & VA periferico & 37 & $54.7(23-74)$ & excluidos & No reportado & $59.5 \%$ \\
\hline
\end{tabular}

tados en promedio antes de las $6 \mathrm{~h}$ a ECMO) por lo que sus resultados son difíciles de extrapolar al resto de los centros ${ }^{40}$. En la Figura 5 se puede ver un algoritmo de decisión para usar como referencia en estos casos.

\section{Puntos clave:}

1. Conexión a ECMO VV o VA.

2. Si la indicacion es sepsis y falla respiratoria catastrofica conexión recomendada es ECMO VV.

3. Si el paciente tiene shock revisar primero la función ventricular.

\section{Reanimación cardiopulmonar extracorpórea}

ECPR (extracorporeal cardiopulmonary resuscitation), el acrónimo por el cual se conoce esta modalidad, es la utilización del soporte ECMO de despliegue rápido, para proporcionar apoyo circulatorio en pacientes que no logran retorno sostenido de la circulación espontánea (ROSC) ${ }^{5}$.

El determinante más importante del resultado, es el tiempo transcurrido entre el inicio del paro hasta el inicio del soporte vital básico (compresiones toráxicas $)^{41,42}$, pero es también, en la práctica, una de las variables más difíciles de determinar en el momento de la decisión. El intervalo des-

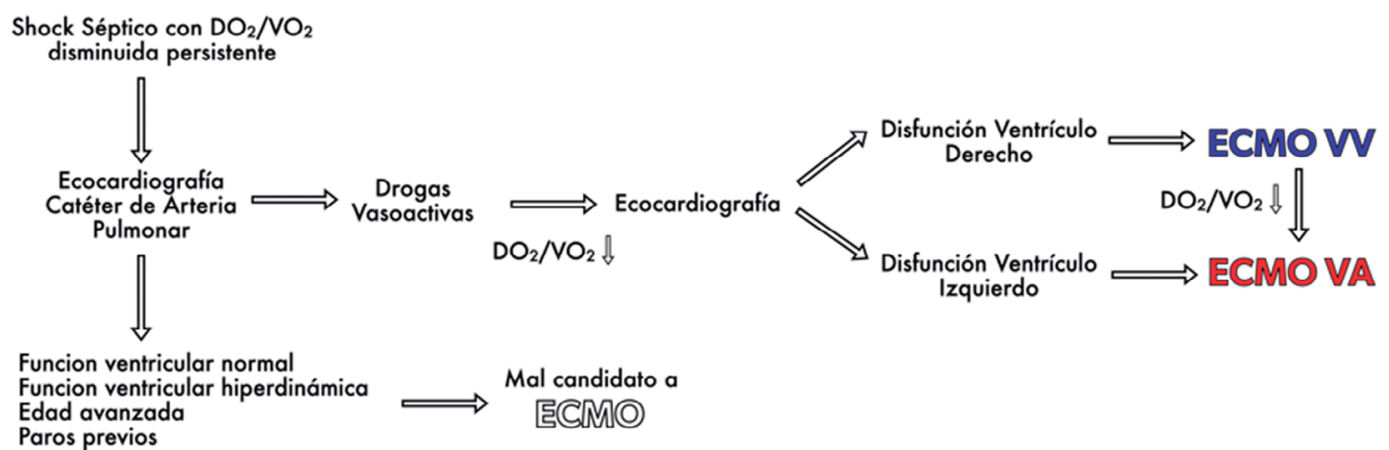

Figura 5. Algoritmo Propuesto ECMO en sepsis (protocolo Clínica Las Condes). 
de el paro hasta el comienzo de la reanimacion cardiopulmonar (RCP) se debe considerar como un período sin flujo, mientras que el tiempo de RCP es un período de bajo flujo, con circulación subóptima $^{43}$ (Figura 6).

La probabilidad de supervivencia con un buen resultado neurológico disminuye rápidamente con cada minuto de RCP convencional, reduciéndose a $<1 \%$ después de 16 min de esta. Es así que despúes de 16-21 min de reanimacion avanzada, se puede plantear que el soporte vital extracorpóreo podria ofrecer una ventaja ${ }^{43,44}$ (Figura 7), con activación y preparación del equipo de ECPR en los primeros 10 min de RCP y canulación dentro de los 30$60 \mathrm{~min}$ posteriores al colapso.

La evidencia en ECPR que existe actualmente es escasa, heterogenea y con manejos no estandarizados, hay en curso estudios aleatorizados en Praga, Paris, Viena, Michigan y Paises Bajos (Registros en https://clinicaltrials.gov/: NCT01511666, NCT02527031, NCT01605409, NCT03065647 y NCT03101787). En lo publicado hasta ahora existe una tendencia a que la sobrevida al alta con buen resultado neurológico aumenta al doble en paro extrahospitalario (OHCA) de adulto (a 20\% aproximadamente $)^{45,46}$. Hay series publicadas de $48 \%$ de sobrevida en OHCA por paro en fibrilación ventricular (FV) refractario definido como la falta de respuesta a $300 \mathrm{mg}$ de amiodarona después de 3 ciclos y manejados inmediatamente llegando al hospital en ECMO, con estudio angiográfico posterior ${ }^{47}$.

En paro cardíaco intrahospitalario (IHCA), en cohortes no emparejadas y estudios con grupos emparejados con puntaje de propensión, se ha encontrado una diferencia significativa en la supervivencia con respecto al alta, 30 días y al año que favorece el ECPR sobre la RCP convencional, con sobrevidas cercanas a $30-40 \%{ }^{48,49}$.

Puntos clave: Conexión VA (usualmente periférica).

1. La sobrevida sin ECMO en paros refractarios es extremadamente baja.

2. Las posibilidades de un buen resultado neurológico disminuyen dramáticamente con el aumento del tiempo de inicio del soporte vital básico, soporte vital avanzado o tiempo a la canulación ${ }^{50}$.

3. La asistolía como ritmo inicial prácticamente no tiene sobrevida ${ }^{51}$.

4. Respete criterios de exclusión ${ }^{52}$.

5. Realizar un "tiempo fuera multidisciplinario" antes de canular, que son 30-60 segundos de una reunion entre el rescatista, lider de la reanimacion, médico ECMO y cardiólogo para decidir si el paciente va a ECPR o no (pausa antes de la decisión).

En la Figura 8, se describen los pasos en un paro extrahospitalario.

\section{ECMO de transporte interhospitalario}

En comparación con el soporte extracorpóreo realizado en un centro ECMO, la instalación, el transporte y la recuperación de pacientes fuera de este lugar (transporte interhospitalario), requiere de esfuerzos logísticos, clínicos y organización adicionales $^{53}$. Esto se hace generalmente en hospitales

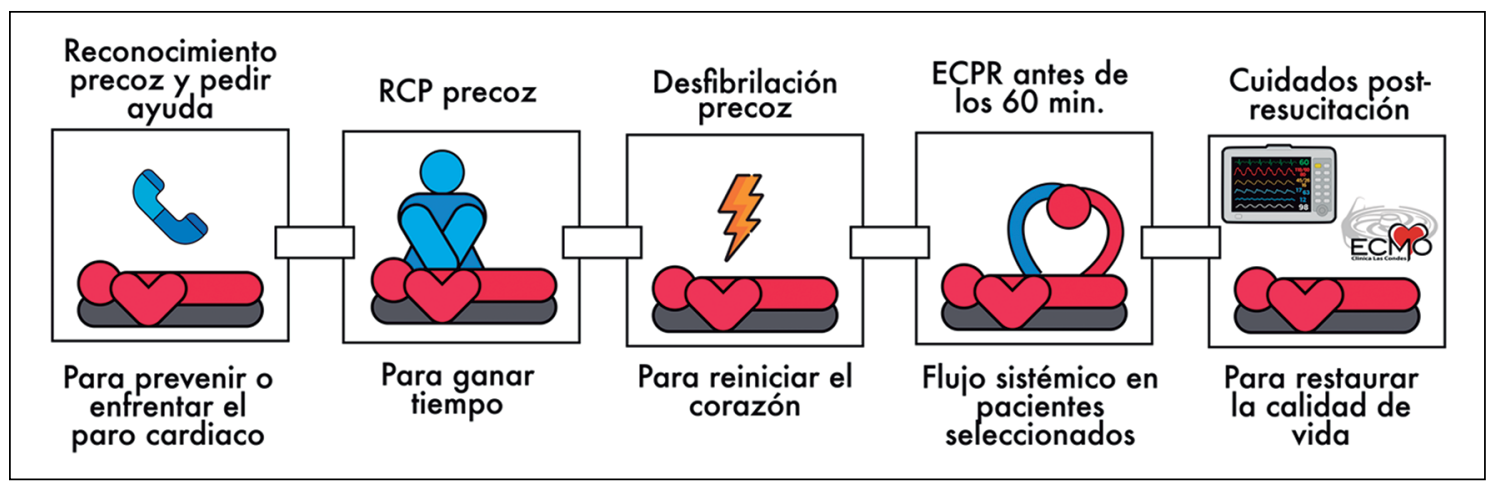

Figura 6. ECMO como un eslabón más en la cadena de supervivencia. RCP: reanimación cardiopulmonar. ECPR: reanimación cardiopulmonar extracorpórea. El resultado depende de las etapas previas, posteriores y una cuidadosa selección. 


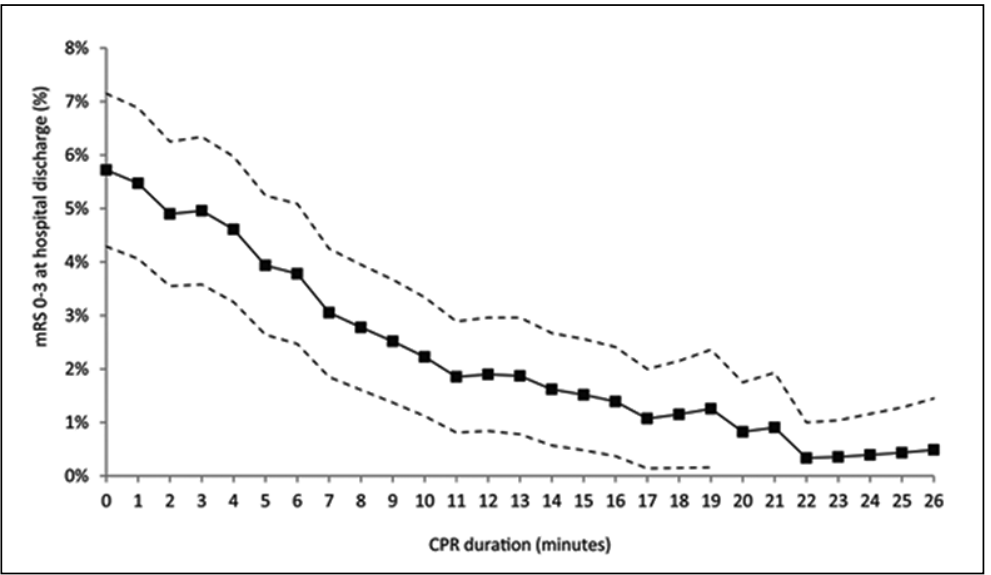

Figura 7. Posibilidad de sobrevida con resultado neurológico vs tiempo de reanimación. Líneas discontinuas: intervalo confianza 95\%. CPR: cardiopulmonary resuscitation; mRS: modified Rankin scale (escala que evalúa resultado neurológico). Reynolds, Joshua C., et al. Duration of resuscitation efforts and functional outcome after out-of-hospital cardiac arrest: when should we change to novel therapies? Circulation 2013; 128 (23): 2488-94. Con permiso: Wolters Kluwer Health, Inc.

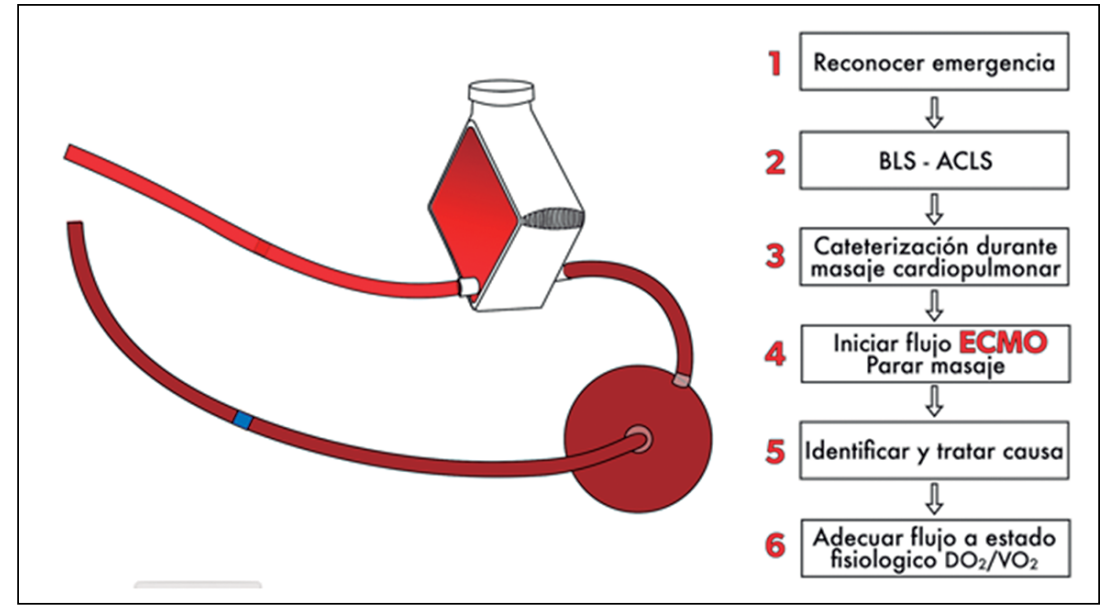

Figura 8. El ECMO como quinto eslabón en la cadena de supervivencia. Importante: pasos en la emergencia de paro cardiaco asociado a resuscitación cardiopulmonar extracorpórea. regionales, que no pueden proporcionar soporte extracorpóreo, seguido del transporte del paciente al centro especializado en $\mathrm{ECMO}^{54}$.

La movilización convencional de un paciente crítico inestable, puede ser peligrosa y mortal, por lo que realizar la canulación en el centro base permite que el traslado sea más seguro ${ }^{55}$.

Otra indicación menos frecuente de transporte, es la necesidad de movilizar a un paciente ya conectado a ECMO, a un centro especializado en otros servicios, como transplante cardiaco y/o pulmonar (transporte secundario).

La elección del medio de transporte esté determinada por la distancia, condiciones climáticas y disponibilidad logística ${ }^{55}$. Independiente del tipo de transporte terrestre o aéreo, estos deben contar con un suministro eléctrico suficiente para la bomba ECMO, el calentador y todos los dispositivos biomédicos necesarios durante el transporte ECMO (Figura 9).

\section{Puntos clave ECMO de Transporte:}

1. Conexión a ECMO VV o VA.

2. Use protocolo y listas de chequeo.

3. Resultados similares a conexión en centro ECMO.

\section{ECMO en pacientes con falla respiratoria catastrofica por coronavirus}

El ECMO ha sido recomendado por la Organización Mundial de la Salud en pacientes con hipoxemia refractaria, pero en centros experimen$\operatorname{tados}^{57}$. Las guías de ELSO enfatizan que el ECMO 


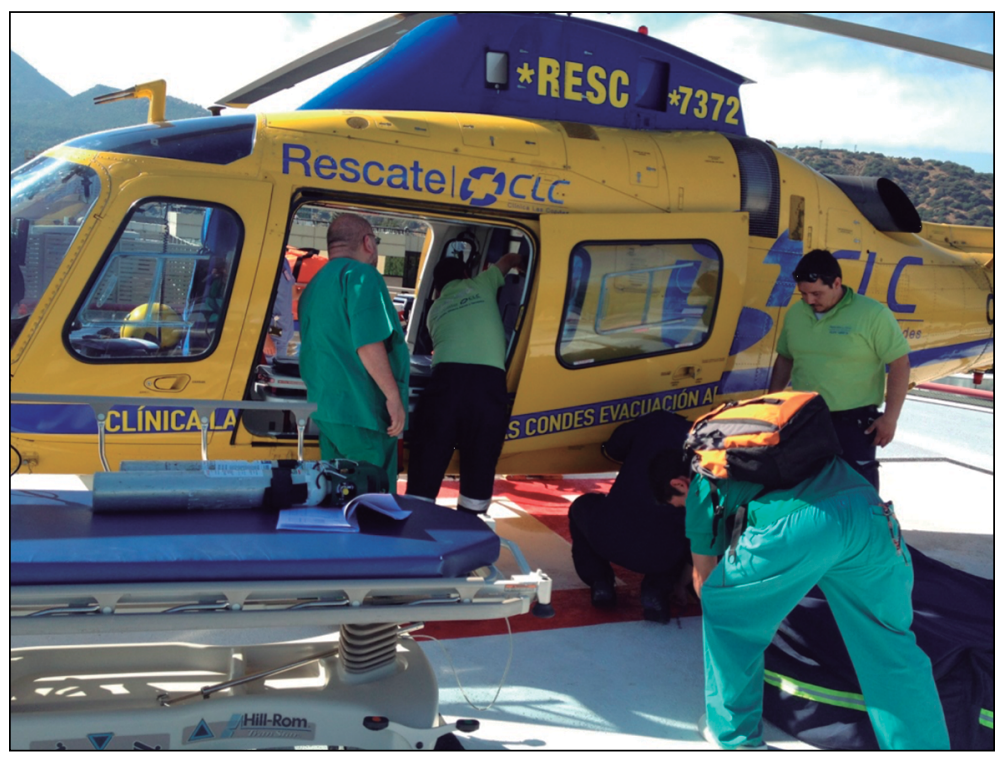

Figura 9.

debe considerarse como una modalidad de apoyo en centros con programas funcionando. Su uso depende mucho de la situación de la pandemia, de la disponibilidad de equipos y recursos humanos, y de los resultados que se vayan publicando, la indicación se ha ajustado a pacientes más bien “jovenes”, sin falla multiorgánica y con menos de 7 -10 dias de ventilación mecánica ${ }^{58,59}$.

En Chile se decidió reforzar los centros ECMO públicos existentes y unificar la información con los centros privados acreditados para esto. $\mathrm{Al} 12 \mathrm{de}$ Abril de 2020 existen 22 cupos en centros privados y 7 en el sistema público (Fuente Minsal) (considerar que esta disponibilidad puede reducirse si los requerimientos de cupos de intensivo se ven sobrepasados). Hasta el 29 de Abril han sido conectados 6 pacientes, tres ya fuera de soporte ECMO y 3 en curso. Se siguen considerando las indicaciones de SOCHIMI MINSAL como referencia, con particular énfasis en prueba de prono previa $^{60}$.

Las indicaciones y contraindicaciones para ECMO durante la pandemia, deben ajustarse periódicamente a factores locales. Las indicaciones en la mayoría de los centros es el criterio EOLIA (Tabla 1). Las comorbilidades del paciente como; edad avanzada, fragilidad, enfermedad pulmonar crónica, diabetes, insuficiencia cardíaca y ventilación mecánica prolongada aumentan significati- vamente el riesgo de mortalidad en la infección grave por coronavirus y, en consecuencia, pueden ser contraindicaciones para $\mathrm{ECMO}^{61}$.

ELSO ha establecido que todos los centros puedan reportar sus casos ECMO en pacientes COVID, siendo parte o no del registro previamente. Es una importante oportunidad de contribuir a los datos y análisis de estos mientras la pandemia está en curso, disponible en: https://www.elso.org/ Registry/FullCOVID19RegistryDashboard.aspx

Puntos clave de ECMO en Falla Respiratoria catastrófica por Coronavirus:

1. Más de $90 \%$ son conexiones a ECMO VV.

2. Su uso dependerá de la situacion de la pandemia versus recursos de cuidados intensivos.

\section{Conclusiones}

ECMO es una tecnología compleja que está conformada por una máquina (hardware) que opera un grupo multidisciplinario (software). Las indicaciones son variadas, pero siempre tienen como objetivo reestablecer la entrega/ demanda de oxigeno y de esta forma obtener tiempo. El entender modos de conexión, manejo de la coagulación, las complicaciones inherentes al sistema, 
el entrenamiento, la capacitación contínua, el soporte institucional y el registro en una base de datos actualizada que permita comparar resultados, son necesarios para el funcionamiento de un programa ECMO.

\section{Referencias}

1. Díaz R, Fajardo C, Rufs J. Historia del ECMO (Oxigenación por membrana extracorpórea o soporte vital extracorpóreo). Rev Med Clin Las Condes 2017; 28 (5): 796-802.

2. Zapol WM, Snider MT, Hill JD, Fallat RJ, Bartlett R, et al. Extracorporeal membrane oxygenation in severe acute respiratory failure: a randomized prospective study. JAMA 1979; 242 (20): 2193-6.

3. Peek GJ, Mugford M, Tiruvoipati R, Wilson A, Allen E, Thalanany MM, et al. Efficacy and economic assessment of conventional ventilatory support versus extracorporeal membrane oxygenation for severe adult respiratory failure (CESAR): a multicenter randomized controlled trial. Lancet 2009; 374 (9698): 1351-63.

4. Conrad SA, Broman LM, Taccone FS, Lorusso R, Malfertheiner MV, Pappalardo F, et al. The extracorporeal life support organization Maastricht treaty for nomenclature in extracorporeal life support. A position paper of the extracorporeal life support organization. Am J Respir Crit Care Med 2018; 198 (4): 447-51.

5. Broman LM, Taccone FS, Lorusso R, Malfertheiner MV, Pappalardo F, Di Nardo M, et al. The ELSO Maastricht Treaty for ECLS Nomenclature: abbreviations for cannulation configuration in extracorporeal life support-a position paper of the Extracorporeal Life Support Organization. Crit Care 2019; 23 (1): 36-43.

6. Ramanathan K, Antognini D, Combes A, Paden M, Zakhary B, Ogino $\mathrm{M}$, et al. Planning and provision of ECMO services for severe ARDS during the COVID-19 pandemic and other outbreaks of emerging infectious diseases. Lancet Respir Med 2020; S2213-2600 (20): 30121-1.

7. Combes A, Hajage D, Capellier G, Demoule A, Lavoué S, Guervilly C, et al. Extracorporeal membrane oxygenation for severe acute respiratory distress syndrome. $\mathrm{N}$ Engl J Med 2018; 378 (21): 1965-75.

8. Hardin C, Hibbert K. ECMO for Severe ARDS. N Engl J Med 2018; 378 (21): 2032-24.

9. Munshi L, Walkey A, Goligher E, Pham T, Uleryk EM, Fan E. Venovenous extracorporeal membrane oxygenation for acute respiratory distress syndrome: a systematic review and meta-analysis. Lancet Respir Med 2019;

\section{7 (2): 163-72.}

10. Abrams D, Ferguson ND, Brochard L, Fan E, Mercat A, Combes A, et al. ECMO for ARDS: from salvage to standard of care? Lancet Respir Med 2019; 7 (2): 108-10.

11. Patroniti N, Zangrillo A, Pappalardo F, Peris A, Cianchi G, Braschi A, et al. The Italian ECMO network experience during the 2009 influenza A (H1N1) pandemic: preparation for severe respiratory emergency outbreaks. Intensive Care Med 2011; 37 (9): 1447-57.

12. Schmidt M, Stewart C, Bailey M, Nieszkowska A, Murphy L, Pilcher D, et al. Mechanical ventilation management during extracorporeal membrane oxygenation for acute respiratory distress syndrome: a retrospective international multicenter study. Crit Care Med 2015; 43 (3): 654-64.

13. Amato MB, Meade MO, Slutsky AS, Brochard L, et al. Driving pressure and survival in the acute respiratory distress syndrome. N Engl J Med 2015; 372 (8): 747-55.

14. Wiedemann HP, Wheeler AP, Bernard GR, Thompson BT, Hayden D, deBoisblanc B, et al; National Heart L, Blood Institute Acute Respiratory Distress Syndrome Clinical Trials N. Comparison of two fluid-management strategies in acute lung injury. N Engl J Med 2006; 354: 2564-75.

15. Papazian L, Forel JM, Gacouin A, Penot-Ragon C, Perrin G, Loundou A, et al; ACURASYS Study Investigators. Neuromuscular blockers in early acute respiratory distress syndrome. N Engl J Med 2010; 363 (12): 1107 16.

16. Guérin C, Reignier J, Richard JC, Beuret P, Gacouin A, Boulain T, et al; PROSEVA Study Group. Prone positioning in severe acute respiratory distress syndrome. N Engl J Med 2013; 368 (23): 2159-68.

17. Schmidt M, Bailey M, Sheldrake J, Hodgson C, Aubron C, Rycus P, et al. Predicting survival after extracorporeal membrane oxygenation for severe acute respiratory failure. The Respiratory Extracorporeal Membrane Oxygenation Survival Prediction (RESP) score. Am J Respir Crit Care Med 2014; 189 (11): 1374-82.

18. Brodie D, Slutsky AS, Combes A. Extracorporeal life support for adults with respiratory failure and related indications: a review. JAMA. 2019; 322(6):557-568.

19. ELSO Adult Cardiac Failure Supplement to the ELSO General Guidelines. Version 1.3. December 2013. Extracorporeal Life Support Organization. Disponible en: https://www.elso.org/Resources/Guidelines.aspx. [Consultado el 1 de marzo de 2020].

20. Agerstrand CL, Bacchetta MD, Brodie D. ECMO for adult respiratory failure: current use and evolving applications. ASAIO J 2014; 60 (3): 255-62.

21. Tipograf Y, Salna M, Minko E, Grogan EL, Agerstrand 
C, Sonett J, et al. Outcomes of Extracorporeal Membrane Oxygenation as a Bridge to Lung Transplantation. Ann Thorac Surg 2019; 107 (5): 1456-63.

22. Abrams D, Brodie D. Extracorporeal membrane oxygenation for adult respiratory failure: 2017 update. Chest 2017; 152 (3): 639-49.

23. Sellers D, Lam K, McRae K. Intraoperative Extracorporeal Life Support for Thoracic and Airway Surgery. In: Slinger P. (eds) Principles and Practice of Anesthesia for Thoracic Surgery. 2019. Springer, Cham.

24. Harjola VP, Lassus J, Sionis A, Køber L, Tarvasmäki T, Spinar J, et al; CardShock Study Investigators; GREAT network. Clinical picture and risk prediction of short term mortality in cardiogenic shock. Eur J Heart Fail 2015; 17 (5): 501-9.

25. Goldberg RJ, Spencer FA, Gore JM, Lessard D, Yarzebski J. Thirty- year trends (1975 to 2005) in the magnitude of, management of, and hospital death rates associated with cardiogenic shock in patients with acute myocardial infarction: a population-based perspective. Circulation 2009; 119: 1211-9.

26. Yip HK, Wu CJ, Chang HW, Chen MC, Hang CL, Fang $\mathrm{CY}$, et al. Comparison of impact of primary percutaneous transluminal coronary angioplasty and primary stenting on short-term mortality in patients with cardiogenic shock and evaluation of prognostic determinants. Am J Cardiol 2001; 87 (10): 1184-8; A4.

27. Baran DA, Grines CL, Bailey S, Burkhoff D, Hall SA, Henry TD, et al. SCAI clinical expert consensus statement on the classification of cardiogenic shock: This document was endorsed by the American College of Cardiology (ACC), the American Heart Association (AHA), the Society of Critical Care Medicine (SCCM), and the Society of Thoracic Surgeons (STS) in April 2019. Catheter Cardiovasc Interv 2019; 94 (1): 29-37.

28. Rossel V, Díaz R, Merello L, Aranguiz-Santander E, Stockins A, Olivares G. Recomendaciones de manejo de pacientes con asistencia circulatoria mecánica de corta duración. Rev Med Chile 2018; 146 (1): 96-106.

29. Ponikowski P, Voors AA, Anker SD, Bueno H, Cleland JG, Coats AJ, et al; Authors/Task Force Members; Document Reviewers. 2016 ESC Guidelines for the diagnosis and treatment of acute and chronic heart failure: The Task Force for the diagnosis and treatment of acute and chronic heart failure of the European Society of Cardiology (ESC). Developed with the special contribution of the Heart Failure Association (HFA) of the ESC. Eur J Heart Fail 2016; 18 (8): 891-975.

30. Levy B, Bastien O, Karim B, Cariou A, Chouihed T, Combes A, et al. Experts' recommendations for the management of adult patients with cardiogenic shock.
Ann Intensive Care 2015; 5 (1): 52.

31. Sheu JJ, Tsai TH, Lee FY, Fang HY, Sun CK, Leu S, et al. Early extracorporeal membrane oxygenator-assisted primary percutaneous coronary intervention improved 30-day clinical outcomes in patients with ST-segment elevation myocardial infarction complicated with profound cardiogenic shock. Crit Care Med 2010; 38 (9): 1810-7.

32. Tehrani BN, Truesdell AG, Sherwood MW, Desai S, Tran HA, Epps KC, et al. Standardized team-based care for cardiogenic shock. J Am Coll Cardiol 2019; 73 (13): 1659-69.

33. Cevasco M, Takayama H, Ando M, Garan AR, Naka $\mathrm{Y}$, Takeda K. Left ventricular distension and venting strategies for patients on venoarterial extracorporeal membrane oxygenation. Journal of thoracic disease 2019; 11 (4): 1676-83.

34. Park TK, Yang JH, Jeon K, Choi SH, Choi JH, Gwon $\mathrm{HC}$, et al. Extracorporeal membrane oxygenation for refractory septic shock in adults. Eur J Cardiothorac Surg 2015; 47 (2): e68-74.

35. Cheng A, Sun HY, Tsai MS, Ko WJ, Tsai PR, Hu FC, et al. Predictors of survival in adults undergoing extracorporeal membrane oxygenation with severe infections. J Thorac Cardiovasc Surg 2016; 152 (6): 1526-1536.e1.

36. Ro SK, Kim WK, Lim JY, Yoo JS, Hong SB, Kim JB. Extracorporeal life support for adults with refractory septic shock. J Thorac Cardiovasc Surg 2018; 156 (3): 1104-9.

37. Huang CT, Tsai YJ, Tsai PR, Ko WJ. Extracorporeal membrane oxygenation resuscitation in adult patients with refractory septic shock. J Thorac Cardiovasc Surg 2013; 146 (5): 1041-6

38. Takauji S, Hayakawa M, Ono K, Makise H. Respiratory extracorporeal membrane oxygenation for severe sepsis and septic shock in adults: a propensity score analysis in a multicenter retrospective observational study. Acute Med Surg 2017; 4 (4): 408-17.

39. Schmidt M, Bréchot N, Combes A. Ten situations in which ECMO is unlikely to be successful. Intensive Care Med 2016; 42 (5): 750-2.

40. Falk L, Hultman J, Broman LM. Extracorporeal membrane oxygenation for septic shock. Crit Care Med 2019; 47 (8): 1097-105.

41. Kurosaki H, Ohta K, Wato Y, Yamashita A, Inaba H. Assessing the efficacy of the new protocol for chest compressions before definitive cardiac arrest in emergency medical service-witnessed adult out-of-hospital cardiac arrests. Resuscitation 2018; 130: 92-8.

42. Tanguay-Rioux X, Grunau B, Neumar R, Tallon J, Boone R, Christenson J. Is initial rhythm in OHCA a predictor of preceding no flow time? Implications for 
bystander response and ECPR candidacy evaluation. Resuscitation 2018; 128: 88-92.

43. Reynolds JC, Frisch A, Rittenberger JC, Callaway CW. Duration of resuscitation efforts and functional outcome after out-of-hospital cardiac arrest: when should we change to novel therapies? Circulation 2013; 128 (23): 2488-94.

44. Kim SJ, Jung JS, Park JH, Park JS, Hong YS, Lee SW. An optimal transition time to extracorporeal cardiopulmonary resuscitation for predicting good neurological outcome in patients with out-of-hospital cardiac arrest: a propensity-matched study. Crit Care 2014; 18 (5): 535.

45. Ortega-Deballon I, Hornby L, Shemie SD, Bhanji F, Guadagno E. Extracorporeal resuscitation for refractory out-of-hospital cardiac arrest in adults: a systematic review of international practices and outcomes. Resuscitation 2016; 101: 12-20.

46. Sakamoto T, Morimura N, Nagao K, Asai Y, Yokota H, Nara S, et al; Group S-JS. Extracorporeal cardiopulmonary resuscitation versus conventional cardiopulmonary resuscitation in adults with out-of-hospital cardiac arrest: a prospective observational study. Resuscitation 2014; 85: 762-8.

47. Bartos JA, Carlson K, Carlson C, Raveendran G, John R, Aufderheide TP, et al. Surviving refractory out-of-hospital ventricular fibrillation cardiac arrest: critical care and extracorporeal membrane oxygenation management. Resuscitation 2018; 132:47-55

48. Chen YS, Lin JW, Yu HY, Ko WJ, Jerng JS, Chang WT, et al. Cardiopulmonary resuscitation with assisted extracorporeal life-support versus conventional cardiopulmonary resuscitation in adults with in-hospital cardiac arrest: an observational study and propensity analysis. Lancet 2008; 372 (9638): 554-61

49. Ouweneel DM, Schotborgh JV, Limpens J, Sjauw KD, Engström AE, Lagrand WK, et al. Extracorporeal life support during cardiac arrest and cardiogenic shock: a systematic review and meta-analysis. Intensive Care Med 2016; 42 (12): 1922-34.

50. Fagnoul, D., Combes, A., \& De Backer, D. Extracorporeal cardiopulmonary resuscitation. Current opinion in critical care 2014, 20(3), 259-265.

51. Dennis M, McCanny P, D'Souza M, Forrest P, Burns $\mathrm{B}$, Lowe DA, et al. Extracorporeal cardiopulmonary resuscitation for refractory cardiac arrest: a multicentre experience. Int J Cardiol 2017; 231: 131-6.

52. Herrada L, Santelices JL, Orrego R, Díaz R. Extracor- poreal cardiopulmonay resuscitation: case report on an out-of-hospital cardiopulmonary arrest. Rev Med Chile 2018; 146 (2): 260-265.

53. Bonadonna D, Barac YD, Ranney DN, Rackley CR, Mumma K, Schroder JN, et al. Interhospital ECMO Transport: Regional Focus. Semin Thorac Cardiovasc Surg 2019; 31 (3): 327-34.

54. Vieira J, Frakes M, Cohen J, Wilcox S. Extracorporeal Membrane Oxygenation in Transport Part 1: Extracorporeal Membrane Oxygenation Configurations and Physiology. Air Med J 2020; 39 (1): 56-63.

55. Grupo de trabajo ECMO de la Sociedad Chilena de Medicina Intensiva. Protocolo operativo para ECMO veno-venoso en falla respiratoria grave. Rev Chil Med Inten 2015; 30: 75-8.

56. Lindén V, Palmér K, Reinhard J, Westman R, Ehrén $\mathrm{H}$, Granholm $\mathrm{T}$, et al. Inter-hospital transportation of patients with severe acute respiratory failure on extracorporeal membrane oxygenation-national and international experience. Intensive Care Med 2001; 27 (10): 1643-8.

57. World Health Organization. Clinical management of severe acute respiratory infection (SARI) when COVID-19 disease is suspected: interim guidance, 13 March 2020. WHO/2019-nCoV/clinical/2020.4. https://apps.who.int/ iris/rest/bitstreams/1272156/retrieve. [Accedido el 12 de abril de 2020].

58. Bartlett RH, Ogino MT, Brodie D, McMullan DM, Lorusso R, MacLaren G, et al. Initial ELSO Guidance Document: ECMO for COVID-19 Patients with Severe Cardiopulmonary Failure. ASAIO J. 2020 Mar. https:// journals.lww.com/asaiojournal/toc/9000/00000. [Accedido el 12 de abril de 2020].

59. Maclaren G, Fisher D, Brodie D. Preparing for the most critically ill patients with COVID-19: the potential role of extracorporeal membrane oxygenation. JAMA 2020 Feb 19.

60. Sociedad Chilena de Medicina Intensiva. Recomendaciones para el manejo de la insuficiencia respiratoria aguda en pacientes con neumonia por coronavirus $\mathrm{V}$. 1.0. https://www.medicina-intensiva.cl/reco/RECOMENDACIONES_MANEJO_INSUFICIENCIA_RESPIRATORIA2020.pdf [Accedido el 13 de abril de 2020].

61. Augoustides JG. Extracorporeal Membrane Oxygenation - Crucial Considerations during the Coronavirus Crisis [published online ahead of print, 2020 Apr 7]. J Cardiothorac Vasc Anesth. 2020. 